Laura Mellini et Francesca Poglia Mileti

\title{
L’agentivité sexuelle au prisme du genre et de la migration : le cas des jeunes issu.e.s de la migration subsaharienne en Suisse
}

Résumé: Cet article, fondé sur les résultats d'une enquête qualitative menée auprès de jeunes issu.e.s de la migration subsaharienne vivant en Suisse, montre que les trajectoires, les représentations et les pratiques sexuelles ne peuvent pas être comprises en dehors des enjeux identitaires liés aux rapports interethniques. Face à une pluralité de normes sexuelles et de références culturelles, religieuses ou morales portées en présence dans des milieux sociaux différents (famille, communauté migrante, groupes de pairs, école, etc.), les jeunes font preuve d'agentivité sexuelle. Cette dernière varie en fonction du genre et de la migration.

Mots-clés: agentivité sexuelle, migration subsaharienne, normes sexuelles, rapports interethniques, socialisation sexuelle

\section{Sexual agency through the lens of gender and migration: The case of sub-Saharan youth in Switzerland}

Summary: Based on the results of a qualitative research conducted with young people from sub-Saharan Africa living in Switzerland, this article shows that sexual trajectories, representations and practices cannot be understood outside of the identity issues related to interethnic relations. Faced with a plurality of sexual norms and cultural, religious or moral references in different social environments (family, migrant community, peer groups, school, etc.), young people demonstrate sexual agency. The latter varies according to gender and migration.

Keywords: sexual agency, sub-Saharan migration, sexual norms, interethnic relations, sexual socialization 
Comme toute socialisation - à savoir l'apprentissage de valeurs et codes sociaux transmis par autrui ou acquis au travers de l'expérimentation - la socialisation sexuelle est un processus biographique, relationnel et identitaire (Dubar, 2002) qui conduit l'individu à avoir des pratiques sexuelles concrètes, mais qui engage aussi tout un ensemble d'éléments émotionnels et culturels. Les normes sexuelles (Bozon \& Rennes, 2015) qui indiquent ce qui est socialement et moralement valorisé en matière de sexualité, pèsent sur les choix individuels et varient en fonction des contextes historiques et sociaux. Le champ du travail social en santé sexuelle ne fait pas exception et constitue donc aussi un espace de production et de négociation des normes sexuelles. Si la prévention des risques constitue historiquement le cœur de l'intervention, les droits sexuels - qui sont reconnus comme droits fondamentaux (Voide-Crettenand, 2017) ${ }^{1}$ sont des références de plus en plus mobilisées dans le domaine de la sexualité. En Suisse, ces droits ne sont pas ancrés directement dans la Constitution fédérale mais figurent indirectement dans plusieurs textes de loi comme, par exemple, les Dispositions du code pénal contre l'atteinte à la vie et à l'intégrité corporelle, la Loi sur l'égalité des sexes, la Loi sur les mutilations génitales féminines (Voide-Crettenand, 2012). Ce cadre légal permet dès lors de légitimer la promotion des principes d'égalité, de diversité et de liberté individuelle en matière de sexualité (Poglia Mileti et al., 2018).

Quels sont les principes transmis au sein des familles issues de la migration? Comment les jeunes font-ils et elles face à des éventuelles injonctions contradictoires et gèrent les différentes normes sexuelles? La gestion de ces tensions normatives est-elle différente selon le genre, dont on connaît l'impact dans le domaine de la sexualité, ou selon que les jeunes aient été (partiellement) socialisé·e·s en Suisse ou au pays d'origine?

Dans cet article, nous présentons les résultats d'une recherche empirique qualitative qui porte sur les trajectoires, les représentations et les pratiques sexuelles des jeunes (18-24 ans) issu·e.s de la migration subsaharienne vivant en Suisse rencontré·e·s durant l'enquête JASS ${ }^{2}$. Nous montrons qu'en réponse aux injonctions contradictoires entre les normes sexuelles portées par les différent·e·s acteur.rice·s qui interviennent au cours de leur socialisation sexuelle (les parents, les ami·e·s, les partenaires, l'école et les professionnel·le·s de la santé), les jeunes doivent faire preuve d'agentivité sexuelle. Cette forme d'agir en contexte, qui conjugue action et responsabilité, se décline différemment selon le genre et la migration. Cette agentivité dépend des valeurs et normes sexuelles acquises au fil de 
la socialisation mais s'inscrit aussi dans des enjeux identitaires liées aux relations interethniques.

\section{Jeunesse, migration et agentivité sexuelle}

Jeunes issu·e.s de la migration et sexualité : un terrain de recherche inexploré

Ce n'est que récemment et assez timidement que les sciences sociales ont, en Suisse, investi le champ de la sexualité ou plutôt celui de la santé sexuelle, car il est vrai que la recherche dans ce domaine est le plus souvent adossée à des problèmes publics liés à la prévention en matière de santé sexuelle (santé reproductive, infections sexuellement transmissibles, etc.). C'est le cas de l'unique enquête nationale menée en 2016 auprès d'environ 30000 personnes par un institut de sondage sur mandat de l'Office fédéral de la santé publique, dans le cadre de la campagne de prévention LOVE LIFE (Hermann et al., 2016). Quant à la sexualité des jeunes, l'enquête de Barrense-Dias et collègues (2019), menée en 2018 auprès de plus de 5000 individus âgés entre 24 et 26 ans, avait pour but d’obtenir des données épidémiologiques sur leur santé sexuelle et reproductive et leurs comportements sexuels. Les résultats montrent que les principales sources d'information dans le domaine de la sexualité sont les ami·e·s (38,9\%), les parents (27,3\%), l'école (19,1\%) et Internet (8\%) (Barrense-Dias et al., 2019). L'étude quantitative et qualitative de Colombo et collègues (2018), quant à elle, est focalisée sur les transactions sexuelles des jeunes, entendues comme des expériences sexuelles associées à un échange financier, matériel et/ou symbolique.

Enfin, les études portant sur la sexualité des personnes issues de la migration disponibles en Suisse sont focalisées sur les expériences des populations en provenance de l'Afrique subsaharienne, autant en termes de problèmes de santé sexuelle, notamment de VIH/sida (Mellini et al., 2018; Villani et al., 2018), que de comportements préventifs ou à risques (Simonson et al., 2015). Le choix de ces populations comme sujets de recherche s'inscrit dans une optique de prévention à la fois primaire et secondaire en matière de santé sexuelle qui est justifiée par des raisons épidémiologiques, puisque c'est dans cette région de l'Afrique que se concentre le plus grand nombre de pays à haute prévalence du VIH/sida. Quant aux jeunes migrant·e·s, l'étude qualitative de Carbajal et collègues (2020) a analysé les prises de risque des jeunes hommes latino-américains dans le cadre de rapports hétérosexuels occasionnels non protégés qui s'inscrivent dans un système hétéronormatif, mettant au centre le plaisir masculin. Ces comportements à risque sont rapportés aussi bien par les jeunes récemment 
arrivé·e·s en Suisse, que par les jeunes issu·e·s de la deuxième génération et les jeunes latino-suisses, descendant.e.s de couples binationaux (Carbajal et al., 2020). L'enquête JASS, contrairement à cette étude, montre que les expériences et les représentations des jeunes subsaharien·ne·s dans le domaine de la santé sexuelle et de la sexualité diffèrent selon qu'ils et elles soient de première ou deuxième génération, puisqu'elles dépendent des contextes dans lesquels les jeunes ont été socialisé·e·s (Mellini et al., 2020; Poglia Mileti et al., 2018, 2019).

\section{Une approche compréhensive sur un sujet sensible}

Les constats et réflexions présentés dans cet article sont basés sur l'analyse de 47 entretiens compréhensifs (Kaufmann, 1996) menés auprès d'hommes et de femmes, originaires d'un pays d'Afrique subsaharienne, âgé·e·s entre 18 et 24 ans et résident.e.s en Suisse romande. D’une durée moyenne de $2 \mathrm{~h} 30$, les entretiens ont été entièrement retranscrits et analysés progressivement, selon les principes de la grounded theory (Corbin \& Strauss, 2008). Choisissant une approche sensible et compréhensive, nous avons développé un dispositif méthodologique axé sur la réflexivité, déjà expérimenté dans une enquête précédente sur les femmes séropositives d'origine subsaharienne (Villani et al., 2014, 2015) avec la rédaction de notes personnelles après chaque entretien ou par le biais de discussions collectives. Pour préparer l'entrée dans le terrain, l'équipe de recherche a organisé un focus group avec 5 expert.e-s travaillant dans les domaines de la santé sexuelle et de la migration et/ou dans des associations africaines. Les échanges ont porté sur les stratégies de recrutement, la relation d'enquête et le guide d'entretien et ont permis de gérer la proximité/distance sociale entre enquêtrices et enquêté·e·s, les éventuels malentendus interculturels pour objectiver l'écart entre enquêtrices et enquêté·e·s (les différences d'âge, de genre, de sexe et d'origine) sur la production des données (Amsellem-Mainguy \& Vuattoux, 2018).

Le choix d'équilibrer la population selon le genre (à signaler que toutes les personnes se sont présentées comme cisgenres) et selon la génération liée à la migration s'est révélé adéquat puisque ces deux dimensions orientent fortement les trajectoires sexuelles. Afin de comprendre si la durée de la présence en Suisse pouvait avoir une incidence sur la socialisation sexuelle, nous avons distingué deux catégories. D'une part, les jeunes de première génération (G1) né·e·s dans un pays d'Afrique subsaharienne qui ont migré après l'âge de 10 ans. D'autre part, les jeunes de deuxième génération (G2) né·e·s en Suisse de parents migrants ou qui sont arrivé·e·s 
Tableau 1:

Caractéristiques des jeunes interviewé·e·s $(\mathrm{N}=47)$

\begin{tabular}{llr}
\hline & & $n$ \\
\hline Genre & femme & 26 \\
& homme & 21 \\
Expérience migratoire & $G_{1}$ & 24 \\
& G2 & 23 \\
Âge & $18-21$ & 23 \\
& $22-25$ & 24
\end{tabular}

Origine

Statut légal

Angola (1), Burundi (2), Cameroun (6), Cap Vert (2), Congo (10), Erythrée (10), Gambie (1), Ghana (1), Guinée (1), Côte d’Ivoire (1), Nigéria (2), Rwanda (2), Sénégal (1), Somalie (3), Soudan (2), Togo (1), Zimbabwe (1)

nationalité

permis $\mathrm{C}$

permis $B$

permis $\mathrm{F}$

permis $\mathrm{N}$

sans papiers

Statut conjugal

en couple

seul.e

Degré de formation

primaire

secondaire I

secondaire II

tertiaire

Statut professionnel

employé.e

sans emploi

apprenti-e

étudiant·e

cours de mise à niveau 5

Religion

catholique

musulmane 9

protestante 8

$\begin{array}{ll}\text { orthodoxe } & 7\end{array}$

évangélique 3

sans 2

$\begin{array}{llr}\text { Initiation sexuelle } & \text { oui } & 37\end{array}$

non 10

$\begin{array}{lll}\text { Âge au premier rapport sexuel } \quad 12-15 & 7\end{array}$

$16-21 \quad 30$

$\begin{array}{lll}\mathrm{Nr} \text { de partenaires sexuel.le·s } & 1-5 & 29\end{array}$

6-15 5

$25-50 \quad 3$

L'enquête a été menée dans le respect des principes éthiques de toute recherche en sciences sociales (Villani et al., 2014, 2015). Les prénoms utilisés dans cet article sont fictifs. 
en Suisse avant l'âge de 10 ans. Les objectifs de l'enquête visaient à étudier la socialisation à la sexualité des jeunes mais aussi les risques face au VIH. Reprendre une catégorie mobilisée dans les approches épidémiologiques à savoir la population issue de l'Afrique subsaharienne qui est une région identifiée comme à forte prévalence du VIH/sida (Poglia Mileti et al., 2019) nous permettait d'apporter un éclairage sociologique et un point de vue émic des personnes qui sont visées par les programmes de prévention spécifiques. Quant à la catégorie de la nationalité, elle n’a pas constitué un critère de sélection de l'échantillon. Elle ne s'est pas révélée pertinente pour expliquer les différences de pratiques et de représentations liées à la sexualité. La population considérée dans cette enquête est une catégorie hétérogène du point de vue de l'origine nationale, des trajectoires scolaires, de la classe sociale d'appartenance, des statuts juridiques, etc. Nous estimons, toutefois, qu'elle a une pertinence sociologique puisque ces jeunes partagent, de fait, l'expérience commune d'être considéré·e·s comme une minorité visible en Suisse. En effet, les représentations et pratiques sexuelles ne se limitent pas aux territoires de l'intime, mais sont fondées et justifiées par des références culturelles acquises au cours de la socialisation. Ces dernières doivent être réélaborées afin de répondre aux enjeux sociaux, symboliques et identitaires liés à la sexualité qui, pour les jeunes rencontrés·e·s, s'inscrivent dans des rapports interethniques: loyauté à l'égard des attentes des parents, respect des principes moraux en vigueur dans la communauté migrante, identification aux pairs, distanciation de pratiques considérées comme non religieuses, etc. (Poglia Mileti et al., 2018).

\section{L'agentivité sexuelle}

Les résultats montrent comment, face à des normes sexuelles différentes (ou même contradictoires), les jeunes font preuve d'agentivité sexuelle. Ce que les anglophones désignent par le terme de sexual agency (Averett et al., 2008; Froyum, 2010; Jewkes \& Morell, 2012; Lesh \& Kruger, 2005) est «la capacité des hommes et des femmes de prendre en charge leur propre sexualité et de l'exprimer de façon positive» (Lang, 2011, p. 189). Elle se traduit par la prise d'initiative, la conscience du désir, la confiance et la liberté de s'engager ou pas dans des comportements sexuels (Averett et al., 2008). La capacité d'agir peut donc être qualifiée de "compétente, raisonnée, consciencieuse et réfléchie» (Lang, 2011, p. 190) soulignant ainsi les dimensions de choix et de responsabilité liées aux pratiques sexuelles. Cette capacité à agir est limitée ou encadrée par les structures sociales (White \& Wyn, 1998) et s'inscrit dans des rapports de genre, de classe et d'ethnicité (Lesch \& Kruger, 2005). 
Les rapports sociaux de sexe régissent fortement les pratiques et les représentations, à l'instar de l'idée reçue selon laquelle les hommes auraient des besoins sexuels naturellement plus importants que les femmes (Bajos et al., 2008). Si cela semble moins évident, l'expérience de la sexualité est aussi encadrée par les rapports de classe. Par exemple, l'homogamie sociale dans le choix des conjoints (Bozon \& Héran, 2006) est toujours d'actualité, comme en atteste une étude récente menée en Suisse (Ravazzini et al., 2017). Il en va de même pour les rapports interethniques qui orientent les représentations réciproques entre groupes, les attentes des familles (Collet \& Santelli, 2012) ou le choix des partenaires, à l'exemple des jeunes migrant·e·s d'origine subsaharienne vivant en Suisse qui, pour la plupart, entrent dans la sexualité avec un·e partenaire de la même origine (Poglia Mileti et al., 2018). Enfin, les rapports entre générations (ou au sein de la même génération) sont à l'œuvre dans le contrôle social sur la sexualité des jeunes (Bozon, 2012; Clair, 2010) comme nous le verrons plus bas.

\section{Les normes sexuelles dans la famille et au sein des groupes de pairs}

Avant de montrer comment le genre et la migration orientent l'agentivité sexuelle, nous présentons les attentes et normes sexuelles privilégiées par les parents et les ami.e.s.

Reproduction des normes sexuelles en famille: quand le genre compte

Qu'ils soient hommes ou femmes, de G1 ou G2, les jeunes rencontré.e.s signalent de manière récurrente l'absence de communication relative au thème de la sexualité au sein de leur famille, estimant qu'elle est considérée comme un «tabou» (Sulstarova et al., 2019) ; le fait de parler de sexualité avec un membre de la famille plus âgé est de surcroît considéré comme un manque de respect à son égard. Cette posture de retrait par rapport à la parole sur la sexualité est un a-priori épistémique dont on ne peut pas faire l'économie, si on s'intéresse à l'intervention dans le domaine de la santé sexuelle et de la sexualité en contexte migratoire.

Lorsque le sujet est tout de même évoqué, les jeunes rapportent que les parents qualifient la sexualité comme quelque chose de «dangereux», "sale» ou "honteux», loin d'une définition de la sexualité fondée sur les droits sexuels ${ }^{3}$, moteurs des programmes d'intervention et de prévention actuels. La dimension de dangerosité est davantage exprimée à l'égard des jeunes femmes interviewées et ce, dès l'arrivée de leurs premières règles, vers l'âge de 10-13 ans, associant pratiques sexuelles, menstruations et idée de souillure (Douglas, 1967). 
Dans ma famille, quand on a les règles, on est une femme, il faut faire attention à son hygiène. Et l'hygiène, ce n'est pas compatible avec les relations sexuelles, ce n'est pas propre. (Anaïs, 21 ans, Angola, G2)

Même si les jeunes ne partagent pas les mêmes catégories morales que les parents en matière de sexualité, il.elle·s ne peuvent pas les ignorer. Les normes sexuelles sont vues par les jeunes comme des particularités culturelles liées à leur origine ou comme des marqueurs identitaires qui permettent de se différencier des pratiques dites occidentales de la sexualité. C'est ainsi que l'agentivité sexuelle des jeunes migrant·e.s se construit en référence à des injonctions familiales qu'il-elle·s contournent ou respectent selon les moments de leur trajectoire sexuelle. Les plus prégnantes sont la norme de la virginité jusqu'au mariage, la norme de l'homogamie ethnique (Santelli \& Collet, 2011) et la norme de l'hétérosexualité. Les jeunes expliquent que ces attentes sociales ont, d'une certaine manière, traversé les pays et les générations pour se reproduire en contexte migratoire. Si l'hétérosexualité est une attente qui vise les deux sexes, les normes de l'homogamie ethnique et de la virginité sont prioritairement adressées aux jeunes femmes. Le contrôle de la sexualité des filles les préserve de l'image de la "fille facile» et montre que les parents assument leur rôle éducatif garantissant la moralité de l'ensemble de la famille (Sulstarova et al., 2019) : la respectabilité des filles est perçue comme le socle de l'honneur familial (Zemmour, 2002). Cet investissement éducationnel a pour but de transmettre les valeurs auxquelles on tient, mais aussi de prouver que l'on est de bons parents aux yeux des membres de la communauté. Concrètement, les interventions des parents, destinées aux jeunes filles et dans une moindre mesure aux jeunes garçons, consistent dans des mises en garde répétées, des interdictions ("pas de sexe avant le mariage») et une surveillance des activités des enfants. Quand les interventions des parents sont adressées aux jeunes garçons, cela advient dans un cadre familial où le référent religieux est important, et ce indépendamment du type de religion (catholique, orthodoxe, protestante ou musulmane).

Ce qu'elle [ma mère] m'a dit, c'est que c'est inutile que tu sortes avec quelqu'un avec qui tu ne vas pas te marier, elle va pas être ta femme. C'est aussi interdit dans notre religion de faire... comment dire...d'avoir des rapports sexuels en dehors [du mariage]. (Heinz, 19 ans, Gambie, G1) 
La transformation des normes sociétales en matière de sexualité tend à créer des divergences de point de vue entre les jeunes et leurs parents (Moguérou et al., 2013) quel que soit le profil familial. Toutefois, à en croire nos répondant.e.s, il existerait des distinctions notables entre leurs parents et ceux de leurs ami-e·s (suisses ou d'autres origines) dans la manière d'aborder la sexualité. Une autre différence réside dans le fait que ne pas respecter les codes sexuels familiaux peur être interprété par leurs parents comme un acte de désaffiliation identitaire. En effet, maintenir certains traits culturels qu'on estime être propres à son origine, permet - dans le cadre de relations interethniques - de marquer les frontières entre les groupes (Barth, 1969) et de maintenir une identité que l'on sent menacée (par une dissolution des références culturelles ou, à l'inverse, par un manque de reconnaissance sociale). En ce sens, avoir des relations sexuelles en dehors du mariage peut être jugé à l'aune des références culturelles ou religieuses de son propre groupe, mais cela peut aussi être considéré comme des pratiques de l'exogroupe duquel on aimerait se distancer (par exemple «les Suisses» ou «les Européens»).

Genre, génération, ethnicité et diversification des normes sexuelles dans les groupes de pairs

L'importance des pairs comme agents de la socialisation à la sexualité n'est plus à démontrer et transcendeles clivages de genre et ethniques, puisqu'elle concerne tant les filles que les garçons (Clair, 2010), tant les étranger·ère·s que les autochtones (Barrense-Dias et al. 2019). Notre enquête confirme le rôle joué par les pairs mais montre qu'il diffère entre les G1 et les G2. Comme nous l'avons restitué ailleurs (Villani et al., 2019), cela tient principalement à la constitution et à l'étendue des réseaux de sociabilité dans lesquels sont inscrit.e.s les un·e.s et les autres.

Dans les groupes composés par des jeunes hommes, de G1 ou de G2, les normes sexuelles valorisées sont celles de l'expérimentation et de la virilité. Ils échangent sur leurs expériences personnelles et explicitent leur point de vue sur le corps des femmes dans un langage qu'ils considèrent comme spécifique à des échanges virils.

Quand on est entre garçons, c'est jamais la même chose que s'il y a une autre fille, même si elle n'a rien à voir. Et s'il y a pas la fille, on va dire "ah tu as vu ses fesses? Tu as vu son corps?» (Grégoire, 23 ans, Ghana, G2) 
Les perceptions des jeunes hommes de G1 et de G2 divergent néanmoins sur la question del'homosexualité. Même siles jeunes hommes de G2 admettent qu'il leur serait difficile d'avouer une orientation non hétérosexuelle, ils discutent de ce thème avec leurs amis. À l'inverse, pour les jeunes de Gl, qui appartiennent plutôt à des réseaux ethnocentrés, la norme prescriptive de l'hétérosexualité exclut à leurs yeux toute autre forme de sexualité pour eux-mêmes mais aussi pour les autres. Les termes évoqués font référence à la religion ("c'est des fils de Satan») ou à des pratiques contraires à la nature («c'est pas naturel»).

Dans les groupes composés de filles et de garçons, les échanges portent sur les goûts et les attentes du sexe opposé, dans le but d'affiner les techniques de séduction. Ni la norme de la virginité, ni celle de l'abstinence ne trouvent leur place dans ce contexte. Alors que les jeunes femmes de G1 restent discrètes sur leurs expériences sexuelles personnelles, les jeunes de G2 mettent en avant le droit à vivre une sexualité choisie et valorisent celle-ci comme partie intégrante de la vie: «sans sexualité, la vie n'a pas de sens». Ce positionnement rend possible la thématisation et la discussion autour du plaisir et du désir, autant masculin que féminin.

Dans les groupes de pairs composés uniquement de jeunes femmes, le clivage entre G1 et G2 est important. Les réseaux de sociabilité sont davantage ethnocentrés que diversifiés pour les jeunes femmes ayant grandi en Afrique, car les amies qu'elles fréquentent font partie de leur communauté. Elles sont plus dans la retenue, disent être gênées de parler de sexualité et avouent avoir peu d'occasions d'avoir des relations sexuelles. Elles privilégient plus fréquemment l'abstinence comparativement à celles de G2 et affirment pour la plupart valoriser les normes de l'homogamie ethnique et l'hétérosexualité transmises par leurs parents. En revanche, les normes sexuelles partagées dans les réseaux de pairs fréquentés par les jeunes femmes de G2 ont tendance à se rapprocher de celles des jeunes hommes: l'expérimentation et les relations sexuelles sans qu'ily ait forcément de sentiments amoureux sont valorisées (Villani et al., 2019).

Quand ces mêmes jeunes femmes fréquentent des ami.e.s qui appartiennent à la "communauté africaine», elles sont plus réticentes à parler de leurs expériences sexuelles, par crainte que des informations les concernant ne circulent dans les réseaux composés de compatriotes et n'arrivent aux oreilles de leurs parents.

Ainsi, l'initiative de s'engager dans des comportements sexuels dépend des configurations relationnelles et répond à des normes sexuelles dont l'interprétation peut varier. Nous montrons dans la prochaine sec- 
tion comment les dimensions de genre et le moment d'arrivée en Suisse orientent fortement l'agentivité sexuelle des jeunes migrant·e·s.

\section{Les stratégies de gestion des tensions normatives}

L'intervention dans le domaine de la sexualité en contexte migratoire ne peut ignorer les préférences morales, religieuses et culturelles, mais devrait aussi tenir compte des enjeux identitaires que soulèvent les choix sexuels et qui s'inscrivent dans le cadre des relations interculturelles et intergénérationnelles. Les jeunes se trouvent face à une pluralité de références et modèles sexuels qu'ils et elles évaluent en fonction de leurs valeurs et de leurs envies personnelles. Ils et elles ne peuvent toutefois pas échapper aux attentes de loyauté de leurs groupes d'appartenance et adoptent des stratégies pour gérer ces décalages normatifs.

Nous présentons ci-dessous 4 types de stratégies qui, chacune à sa manière, permettent aux jeunes de ne pas enfreindre (en tout cas ouvertement) les principes moraux transmis par leurs parents tout en vivant leur sexualité en fonction de leurs valeurs.

\section{Les stratégies de dissimulation et d'évitement}

Les stratégies de dissimulation et d'évitement consistent à cacher aux parents, à la famille élargie ou aux membres de sa communauté les indices qui pourraient révéler le fait que l'on a une vie sexuelle active. La pression à laquelle sont soumises les jeunes femmes est importante, puisque des écarts sexuels risquent de tacher l'honneur familial qui dépend de la « réputation» dont elles jouissent dans leur communauté (Poglia Mileti et al., 2018). L'agentivité dont font preuve les jeunes femmes se traduit par une gestion de l'information clivée quant à leur vie sexuelle (ce qui par ailleurs n'est pas inhabituel dans ce domaine). En famille, il s'agit d'éviter de parler de sexualité afin d'échapper à des éventuelles questions sur sa vie intime. Et si les parents devaient en parler, il s'agit de couper court, ne donner aucune information personnelle et orienter la discussion sur d'autres thématiques. Elles n'en parlent même pas avec leurs amies proches ou celles qu'elles appellent les «cousines africaines», pour ne pas décevoir leurs parents qui pourraient découvrir leur sexualité.

Dans le registre de l'action, nous retrouvons plusieurs stratégies comme par exemple le fait d'éviter l'achat de préservatifs dans des magasins ou pharmacies de quartier ou loin de leur domicile ou de demander à des ami-e·s de les leur acheter, par crainte de rencontrer des personnes proches. En tous les cas, ils sont enlevés de la vue de leurs parents. 
Quand on nous avait donné les premiers préservatifs à l'école [dans des cours d'éducation sexuelle], moi je sais que j'avais tout de suite donné à quelqu'un, parce que ma mère ne pouvait pas voir un préservatif dans ma chambre, parce que sinon ça aurait été la catastrophe! (Emma, 25 ans, Cap Vert, G2)

Cette stratégie implique des actions raisonnées, afin de dénicher des endroits discrets pour cacher les préservatifs ou les contraceptifs oraux, le but étant d'échapper au contrôle des parents, rapportés par les jeunes rencontré·e.s.

Après ma maman comme elle fouille un peu partout, elle est allée ranger ma chambre et puis elle est tombée sur plein de choses. Puis, bah ça lui a fait beaucoup de peine d'apprendre que j'avais déjà des relations sexuelles alors qu'elle pensait que j'en avais pas. (Anaïs, 21 ans, Angola, G2)

Au lieu de taire et cacher les signes de leur vie sexuelle active, les jeunes peuvent choisir de les couvrir par des signes d'activités qui, selon leur perception, rencontrent moins de désapprobation parentale. C'est par exemple le cas lorsqu'ils et elles justifient des sorties chez des ami.e.s au lieu d'admettre rencontrer leurs partenaires sexuel-le·s, cela apparaissant comme plus acceptable aux yeux de leurs parents. C'est la même logique qui est à l'œuvre lorsque les jeunes femmes invoquent des contrôles gynécologiques généraux lors de visites médicales pour obtenir un contraceptif ou aborder d'autres problèmes liés à leur activité sexuelle.

D’autres stratégies se lisent dans le choix des partenaires sexuels, notamment du côté des jeunes femmes rencontrées. Certaines évitent de les choisir trop près du domicile parental. D'autres, notamment les jeunes femmes qui entretiennent des relations désengagées sur le plan affectif, qu'elles désignent comme des "plan culs répétés» ou des "coups d'un soir» (Mellini et al., 2020), choisissent des partenaires sexuels d'une autre origine ethnique. Cela leur permet de réduire les risques que leurs parents, par le biais d'autres membres de la communauté, apprennent qu'elles ont une vie sexuelle active, ce d'autant plus sous cette forme.

\section{Stratégies de justifications morales}

Un autre type de stratégies pour gérer le décalage normatif entre les injonctions parentales et celles des pairs ou des partenaires qui poussent au passage à l'acte, revient à adopter des stratégies de justifications morales. Celles-ci peuvent consister à faire coïncider l'entrée dans la sexualité avec 
l'entrée dans l'âge de la majorité légale. Tout se passe comme si le fait d'avoir attendu la majorité pour avoir ses premiers rapports sexuels était interprété comme une moindre dérogation aux normes sexuelles transmises par les parents. Ce faisant, les jeunes s'émancipent d'une obligation parentale mais estiment rester fidèles à l'esprit de la règle (et indirectement à leur culture ou leur communauté) en ayant attendu d'avoir 18 ans pour vivre leurs premières expériences sexuelles.

Alternativement ou conjointement, ces stratégies peuvent amener les jeunes à situer les rapports sexuels dans un contexte relationnel qu'ils et elles jugent moralement approprié (parce que l'on est en couple, parce que l'on connait la personne depuis longtemps, etc.), ce qui les décharge d'un sentiment de trahison à l'égard des parents. C'est le cas d'une fille qui se définit comme musulmane pratiquante et qui, pour pouvoir avoir une sexualité active, s'engage dans des fiançailles «halal».

Si quelqu'un me plait énormément, je vais faire les fiançailles, sans se marier. C'est le halal, c'est quelque chose de religieux. On va voir un sheer [pur]... il y en a dans toutes les mosquées, c'est quelqu'un qui bénit les jeunes sans vraiment les marier. (Mimi, 19 ans, Somalie, G1)

Ces "fiançailles halal» sont une couverture morale et religieuse à une possible vie sexuelle. Elles rappellent le mariage halal étudié par les sociologues françaises, qui est considéré comme une synthèse originale entre, d'une part, les prescriptions matrimoniales héritées de la société d'origine, et transmises dans le cadre familial, et d'autre part, les aspirations plus individualistes des futurs conjoints socialisés en France (Collet \& Santelli, 2012, p. 83). Ces stratégies permettent aux aspirations sexuelles des jeunes femmes de se concrétiser, car si celles-ci outrepassent les prescriptions familiales, elles respectent néanmoins les exigences d'une approbation morale par l'instance religieuse.

Une dernière manière de concilier les attentes parentales et les pressions des pairs consiste à hiérarchiser les pratiques sexuelles et se distancier de celles considérées comme amorales (la multiplication des partenaires sexuel.le-s en dehors des relations amoureuses). Cette stratégie est plus fréquente chez les jeunes filles de G1. Soit elles sont abstinentes, soit elles assument une sexualité qu'elles jugent différente de celle des jeunes femmes ayant grandi en Suisse et/ou ne respectant pas les codes moraux ou religieux qu'elles considèrent comme attendus. 
Comme celle qui boit de l'alcool et sort en culotte, avec des seins presque moitié nus [...] pour moi elle est pas vraiment une musulmane... (Emilie, 23 ans, Côte d'Ivoire, G1)

Être respectable du point de vue moral ou religieux signifie adopter des comportements qui interdisent l'affichage en public de corps sexués et qui, idéalement, circonscrivent la sexualité dans le cadre de relations de couple stables orientées vers le mariage. Mais le message religieux a aussi une dimension identitaire, il est utilisé ici comme contre-norme occidentale à une sexualité perçue comme trop libérée (Villani et al., 2019).

\section{Agentivité sexuelle ou quand le genre se conjugue à l'expérience migratoire}

L'agentivité sexuelle, dont les jeunes sont appelé·e·s à faire preuve face à la pluralité des normes, prend des formes différentes. L'analyse des entretiens montre que le genre et le moment de leur arrivée en Suisse sont deux dimensions qui structurent les choix, représentations et pratiques au fondement de l'agentivité sexuelle. Nous présentons ci-dessous 4 types d'agentivité liées au genre et au moment de la migration.

\section{L'agentivité des jeunes hommes}

Les jeunes hommes de G1, indépendamment de leur pays d'origine, décrivent un contexte familial dans lequel le thème de la sexualité est considéré comme «tabou». Pour les personnes qui ont subi une circoncision, la communication est réduite à quelques brefs échanges au cours desquels les parents se sont limités à donner des informations pratiques sur l'intervention. Les informations relatives aux risques sexuels et aux pratiques préventives ont été transmises dans le cadre de l'école, pour les jeunes ayant une scolarité secondaire ou supérieure, et des campagnes gouvernementales ayant eu lieu dans le pays d'origine. Certains pays sont décrits comme plus engagés dans la lutte contre le sida, dont le Burundi ou la Côte d'Ivoire, en comparaison à d'autres pays tels que l'Erythrée. Les jeunes hommes qui ont habité en milieu urbain en Afrique disent avoir eu plus facilement accès à ces informations que les jeunes résidant à la campagne, qui n’ont pas fréquenté l'école régulièrement.

Là où j'habitais c'était un village où... il y a des gens qui ne croient pas. Ils trouvent ça fou de prendre du plastique de le mettre sur ton sexe, ils trouvent ça fou! Il y a certains qui n'utilisent pas du tout. Mais bon... c'est surtout les 
étudiants qui utilisent, mais les autres qui n'ont pas vu l'école, non... (David, 19 ans, Sénégal, G1)

Lorsque les jeunes hommes de G1 arrivent en Suisse, ils ont difficilement accès aux institutions de santé sexuelle. N'étant par ailleurs pas toujours insérés dans des cursus scolaires dits ordinaires, ils ont plus rarement l'opportunité de suivre les cours d'éducation sexuelle. Les jeunes requérant·e·s d'asile mentionnent avoir assisté à quelques interventions ponctuelles dans ce domaine, sans pour autant pouvoir en rendre compte aux enquêtrices. Cela limite les informations médicales sur les maladies sexuellement transmissibles (Poglia Mileti et al., 2019).

Les jeunes hommes de Gl affirment avoir plus de libertés que dans leur pays d'origine et peuvent de ce fait faire preuve d'agentivité sexuelle, même si elle reste limitée. En effet, les réseaux de sociabilité sont plutôt restreints et ethnocentrés, notamment pour les mineurs non accompagnés, confinés dans les foyers d'hébergements collectifs. Les liens peu étendus limitent aussi les occasions d'avoir des relations sexuelles (les rapports sexuels sont ponctuels, vécus dans le cadre de relations plutôt occasionnelles) et rendent également difficile le partage d'expériences autour de leur sexualité avec les pairs. Cela ne leur permet pas d'acquérir les codes de la communication sexuelle telle qu'elle est adoptée par les autres jeunes nés en Suisse (Villani et al., 2019).

Les jeunes hommes de G2, quant à eux font preuve d'une agentivité sexuelle qui se présente sous des formes variées. Si, dans le cadre familial le «tabou» sur la sexualité ne semble pas s'estomper, les groupes de pairs et l'école sont deux contextes de socialisation sexuelle dans lesquels les jeunes hommes acquièrent des connaissances, donnent et reçoivent des conseils, échangent leurs expériences. Dans les groupes de pairs, on l'a vu, c'est surtout l'expérimentation qui est valorisée. Dans le cadre scolaire, les jeunes rencontrés estiment que l'accent était mis en priorité sur la prévention avec une approche de la sexualité médicalisée, mais que les concepts de droits sexuels, consentement et égalité ne sont toutefois pas absents des discours des intervenant.e.s.

Sur le plan des pratiques sexuelles, les différents messages que les jeunes entendent dans ces espaces de socialisation se traduisent par une diversification des trajectoires sexuelles. Certains jeunes présentent des trajectoires basées sur l'expérimentation, le détachement affectif et le plaisir (Mellini et al., 2020), alors que d'autres, notamment ceux qui s'identifient comme pratiquants sur le plan religieux, circonscrivent les rapports 
sexuels dans le cadre de relations de couple qu'ils tiennent à décrire comme égalitaires.

\section{L'agentivité sexuelle des jeunes femmes}

Les jeunes femmes de G1 n'ont pas eu l'occasion de parler de sexualité au pays d'origine (faible niveau de scolarisation et absence de discussions en famille) et disent être confrontées, depuis l'enfance, à des cadres moraux où la place de la femme est subsumée à la décision des hommes. En Suisse, elles constatent qu'il y a des espaces où on parle de sexualité, que ce soit dans les groupes de pairs, à l'école ou dans les familles de leurs ami·e·s non issu.e.s de la migration africaine. Certaines se disent «choquées» de ces discours qui explicitent les pratiques intimes car elles n’ont pas été socialisées à la parole sur la sexualité.

À cela, vient s'ajouter une agentivité limitée qui se traduit par une difficulté à négocier sa place au sein des relations intimes. Les pratiques sexuelles sont justifiées par un rapport de genre qui valorise la satisfaction du plaisir masculin ("c'est l'homme qui décide tout»). Le choix de la contraception ou de la protection face aux maladies sexuellement transmissibles est souvent laissé aux partenaires masculins, car «ce sont les hommes qui portent les préservatifs». L'agentivité sexuelle se traduit par une mise à distance du monde intime et par des trajectoires qui sont marquées par l'abstinence ${ }^{4}$ ou une entrée dans la sexualité qui se fait à un âge plus avancé que les pairs vivant dans le même contexte social ${ }^{5}$. La justification donnée par ces jeunes femmes est morale et vise à se distancier de «certaines filles» qui vivent dans une liberté sexuelle qui ne correspond pas à leurs valeurs.

Àl'inverse, l'agentivité sexuelle des jeunes femmes de G2 est la plupart du temps assumée, portée par une démarche d'expérimentation, avec une valorisation du désir et du plaisir dans des relations où l'attachement affectif n'est pas une condition nécessaire. Enfin, cette agentivité se matérialise par des actions de préservation de leur santé sexuelle, comme le fait de se munir de préservatifs quand elles sortent, de refuser les rapports non protégés et d’imposer le port du préservatif à leurs partenaires.

Et puis, ben, moi je lui avais dit que de toute façon je ne couchais pas sans préservatif, sans capote, quoi: ça, c'était exclu, exclu. Tant que moi... pour moi, tant que j'ai pas la feuille de test devant les yeux que t'as pas ci, t'as pas ça comme maladie, moi je couche pas sans capote. (Mégane, 19 ans, Cameroun, G2) 


\section{Discussion conclusive}

Les jeunes issu.e.s de la migration subsaharienne et vivant en Suisse sont confronté·e·s à une pluralité de normes sexuelles et à des injonctions différentes, quand elles ne sont pas divergentes. Pour avoir une vie sexuelle active, sans pour autant enfreindre les injonctions parentales ou la loyauté familiale, ces jeunes sont appelé·e·s à faire preuve d'agentivité sexuelle. Celle-ci consiste en un ensemble de responsabilités, d'actions et de stratégies qui se déclinent selon le genre et la migration. Les jeunes femmes et les jeunes hommes né·e·s en Afrique ou arrivé·e·s après l'âge de 10 ans (G1) sont, de manière générale, moins habitué·e·s à parler de sexualité et ont moins d'opportunités d'avoir des rapports sexuels car leurs réseaux de sociabilité sont plus restreints. En effet, en ligne avec les résultats issus de l'enquête de Barrense-Dias et collègues (2019) sur la sexualité des jeunes en Suisse, les analyses menées ici indiquent que les principales sources d'information et d'échange sur la sexualité sont les ami·e·s. Les échanges avec les parents, en revanche, sont ici beaucoup moins présents que dans l'enquête citée, car la sexualité est considérée un sujet tabou dans les familles concernées. Néanmoins, quand le sujet est abordé, ce sont les mères qui s'en chargent, pour dissuader leurs filles d'avoir des rapports sexuels en dehors du cadre matrimonial, plus que pour faire de la prévention.

Quant aux différences et hiérarchies fondées sur les rapports de genre, elles existent et sont fortes. Si les attentes de genre dans le domaine de la sexualité ne sont évidemment pas uniquement réservées aux femmes issues de la migration (Colombo et al., 2017), ces dernières vivent néanmoins des situations spécifiques. Les jeunes femmes que nous avons rencontrées doivent se positionner par rapports aux valeurs et normes sexuelles transmises par leurs parents et valorisées dans leur communauté (parfois en les contrant ou les détournant) tout en ne reniant pas leurs appartenances. Si les différences de genre tendent à s'atténuer au fil des générations de migrant·e·s, il en reste pas moins que les jeunes femmes de G2 doivent opérer un travail stratégique important pour vivre leur sexualité. En effet, socialisées depuis plus longtemps en Suisse, et conscientes de l'autonomie sexuelle qui leur est laissée dans ce contexte, elles se doivent d'adopter des stratégies sexuelles qui sont en cohérence avec leurs valeurs, même si celles-ci vont à l'encontre des attentes des parents et des représentations des membres de leur communauté. Cela corrobore par ailleurs les résultats des études menées en France sur les descendant·e·s de personnes migrantes (Hamel, 2006). 
Nos résultats montrent donc que la lecture des trajectoires des personnes issues de la migration en termes culturels doit être nuancée. Certes, les représentations et pratiques sexuelles sont encadrées par les normes sexuelles acquises au cours de la socialisation, mais elles ne peuvent pas être comprises en dehors des enjeux moraux et identitaires découlant des rapports d'altérité socialement situés. Ainsi, l'autonomie individuelle en matière de sexualité promue par les droits sexuels, au fondement du travail social, doit être comprise pour les jeunes issu.e.s de la migration comme l'expression d'une agentivité, toujours en prise avec les rapports interethniques.

\section{Références bibliographiques}

Amsellem-Mainguy, Yaëlle \& Vuattoux, Arthur (2018). Enquêter sur la jeunesse. Outils, pratiques d'enquête, analyses. Armand Colin.

Averett, Paige, Benson, Mark \& Vaillancourt, Kourtney (2008). Young women's struggle for sexual agency: the role of parental messages. Journal of Gender Studies, 17(4), 331-344.

Bajos, Nathalie, Ferrand, Michèle \& Andro, Armelle (2008). La sexualité à l'épreuve de l'égalité. In Nathalie Bajos (Eds.), Enquête sur la sexualité en France: Pratiques, genre et santé, p. 545-576. La Découverte.

Bajos, Nathalie, Rahib, Delphine \& Lydié, Nathalie (2018). Genre et sexualité. D'une décennie à l'autre. Baromètre santé 2016. Santé publique France.

Barrense-Dias, Yara, Akre, Christina, Surís, Joan-Charles, Berchtold, André, Morselli, Davide, Jacot-Descombes, Caroline \& Leeners, Brigitte (2019). Does the primary resource of sex education matter? A Swiss national study, The Journal of Sex Research, 57(2), 166-176.

Barth, Fredrik (1969). Ethnic Groups and Boundaries: The social organization of culture difference. George Allen \& Uwin. Bozon, Michel \& Héran, François (2006). La formation du couple. Textes essentiels pour la sociologie de la famille. La Découverte.
Bozon, Michel (2012). Autonomie sexuelle des jeunes et panique morale des adultes: Le garçon sans frein et la fille responsable. Agora débats/jeunesses, 60(1), 121-134.

Bozon, Michel \& Rennes, Juliette (2015). Histoire des normes sexuelles: l'emprise de l'âge et du genre, Clio. Femmes, Genre, Histoire, 42. Récupére de http://journals. openedition.org/clio/12823

Carbajal, Myrian, Ward, Lydia \& Schönenberger, Silvia (2020). Rapports hétérosexuels occasionnels non protégés des jeunes latino-américain·e-s en Suisse: au-delà de la prise de risque. Agora/ débats jeunesse, 84(1), 7-21.

Clair, Isabelle (2010). Des filles en liberté surveillée, dans les espaces ruraux et périurbains aujourd'hui. In Véronique Blanchard (Eds.), Les jeunes et la sexualité: Initiations, interdits, identités (XIX ${ }^{e}-$ XXI siècle), p. 321-329. Autrement.

Collet, Beate \& Santelli, Emmanuelle (2012). Le mariage «halal», réinterprétation des rites du mariage musulman dans le contexte post-migratoire français. Recherches familiales, 9, 83-92.

Colombo, Annamaria, Carbajal, Myrian, Carvalho Barbosa, Marlène \& Tadorian, Marc (2017). Gagner la reconnaissance des pairs en évitant la réputation de "pute». L'injonction paradoxale qui pèse sur les filles impliquées dans des transactions sexuelles, Revue Jeunes et sociétés, 2 (2). http://rjs.inrs.ca/index.php/rjs/ article/view/117 
Corbin, Julien \& Strauss, Anselm (2008). Basics of qualitative research: techniques and procedures for developing grounded theory ( $3^{\text {rd }}$ ed.). Sage.

Douglas, Mary (1967). Purity and Danger. Routledge and Kegan Paul Ltd.

Dubar, Claude (2002). La socialisation. Armand Colin.

Froyum, Carissa M. (2010). Making "good girls": sexual agency in the sexuality education of low-income black girls. Culture, Health \& Sexuality, 12(1), 59-72.

Hamel, Christelle (2006). La sexualité entre sexisme et racisme : les descendantes de migrant.e.s du Maghreb et la virginité, Nouvelles Questions Féministes, 25(1), 41-58.

Hermann, Michael, Bosshardt, Lorenz, Milic, Thomas \& Nowak, Mario (2016). Le sexe en Suisse - une étude de l'institut de sondage sotomo réalisée dans le cadre de la campagne LOVE LIFE. OFSP.

Jewkes, Rachel \& Morrell, Rebecca (2012). Sexuality and the limits of agency among South African teenage women: Theorising femininities and their connections to HIV risk practices. Social Science \& Medicine, 74, 1729-1737.

Kaufmann, Jean-Claude (1996). L'entretien compréhensif. Armand Colin.

Lang, Marie-Eve (2011). L’«agentivitié sexuelle» des adolescentes et des jeunes femmes: une définition. Recherches féministes, 24(2), 189-209.

Lesch, Elmien \& Kruger, Lou-Marie (2005). Mothers, daughters and sexual agency in one low-income South African community. Social Science \& Medicine, 61, 1072-1082.

Marsicano, Élise, Lydié, Nathalie \& Bajos, Nathalie (2011). Genre et migration: l'entrée dans la sexualité et migrants d'Afrique subsaharienne en France. Population, 66(2), 313-341.

Mellini, Laura, Poglia Mileti, Francesca \& Villani, Michela (2018). Résistantes face à la vulnérabilité: l'agentivité relationnelle des femmes africaines et séropositives en contexte migratoire, Terrains \& Travaux, 33(2), 153-174.
Mellini, Laura, Poglia Mileti, Francesca, Sulstarova, Brikela, Villani, Michela \& Singy, Pascal (2020). HIV sexual risk behaviors and intimate relationships among young sub-Saharan African immigrants in Switzerland: A brief report. International Journal of Sexual Health, 32(1), 33-39.

Moguérou, Laure, Hamel, Christelle \& Santelli, Emmanuelle (2013). Genre, origine et autonomisation durant la période de la jeunesse. Les relations entre parents et enfants dans les familles immigrées. Migrations Société, 147-148(3), 189-204.

Poglia Mileti, Francesca, Mellini, Laura, Sulstarova, Brikela \& Villani, Michela (2018). Protection descendante, autoprotection et protection ascendante: familles issues de la migration subsaharienne en Suisse et gestion des risques sexuels. Revue suisse de travail social, 24(2), 15-33.

Poglia Mileti, Francesca, Mellini, Laura, Sulstarova, Brikela \& Villani, Michela (2019). Exploring barriers to consistent condom use among sub-Saharan African young immigrants in Switzerland, Aids Care, 31(1), 113-116.

Ravazzini, Laura, Kuhn, Ursina \& Suter, Christian (2017). Do opposites attract? Educational assortative mating and dynamics of wage homogamy in Switzerland, 1992-2014. Swiss Journal of Sociology, 43(3), 567-586.

Santelli, Emmanuelle \& Collet, Beate (2011). De l'endogamie à l'homogamie socio-ethnique. Réinterprétations normatives et réalités conjugales des descendants d'immigrés maghrébins, turcs et africains sahéliens. Sociologie et sociétés, 43(2), 329-354.

Simonson, Thomas, Dubois-Arber, Françoise, Jeannin, André, Bodenmann, Patrick \& Bize, Raphaël (2015). Comportements face au VIH/sida parmi les migrants originaires d'Afrique subsaharienne en Suisse. Enquête ANSWER 2013-2014, Raisons de santé, 248. Lausanne: IUMSP. 
Sulstarova Brikela, Poglia Mileti Francesca, Mellini Laura, Villani Michela \& Singy Pascal (2019). Parler de sexualité: le point de vue des jeunes migrant.e.s subsaharien·ne.s. Actes du colloque de la Société Internationale de Linguistique Fonctionnelle (SILF), Moscow City University, 121-124.

Villani, Michela, Poglia Mileti, Francesca, Mellini, Laura, Sulstarova, Brikela \& Singy, Pascal (2014). Les émotions au travail (scientifique) : enjeux éthiques et stratégies méthodologiques d'une enquête en terrain intime, Genre, Sexualité \& Société, 12. Récupére de http://gss. revues.org/3333

Villani, Michela, Poglia Mileti, Francesca, Mellini, Laura, Sulstarova, Brikela \& Singy, Pascal (2015). L'engagement (scientifique) sensible: stratégies d'enquête sur les thèmes de la sexualité, la séropositivité et le secret, Civilisations, 64(1), 45-56.

Villani Michela, Mellini Laura et Poglia Mileti Francesca (2018). Femmes subsahariennes séropositives en Suisse: le
VIH à l'épreuve de l'intersectionnalité, Les Cahiers du genre, 65(2), 215-230.

Villani, Michela, Poglia Mileti, Francesca, Mellini, Laura \& Sulstarova, Brikela (2019). Socialisation des jeunes issus des migrations subsahariennes en Suisse, Anthropologie et Développement, 50, 13-31.

Voide Crettenand, Gilberte (2012). Dix droits sexuels fondamentaux. Reiso, Récupéré de https://reiso.org/articles/themes/ ethique/74-dixdroitssexuelsfondamen taux

Voide Crettenand, Gilberte (2017). Les droits sexuels dans l'éducation informelle. Reiso, Récupéré de https://reiso.org/articles/ themes/politiques/1420-les-droitssexuels-dans-l-education-informelle

White, Rob \& Wyn, Johanna (1998). Youth agency and social context. Journal of Sociology, 34(3), 314-327.

Zemmour, Zine-Eddine (2002). Jeune fille, famille et virginité. Approche anthropologique de la tradition, Confluences Méditerranée, 41(2), 65-76.

\section{Annotations}

1 International Planned Parenthood Federation (IPPF), Londres, 2008.

2 JASS, «Jeunes migrant·e·s d'Afrique subsaharienne et santé sexuelle», est une enquête qualitative, financée par le Fonds national suisse de la recherche scientifique (subside n 100017_162382), menée entre 2016 et 2018 par une équipe de sociologues et de sociolinguistes des universités de Fribourg et Lausanne: Francesca Poglia Mileti, Pascal Singy, Laura Mellini, Michela Villani et Brikela Sulstarova.

3 http://www.euro.who.int/fr/health-topics/Life-stages/sexual-and-reproductive-health/news/news/2011/06/sexualhealth-throughout-life/definition

4 Sur les 6 jeunes femmes non initiées à la sexualité pénétrative, 5 femmes sont de G1.
5 Certains auteur.e.s utilisent le terme d'entrée dite tardive dans la sexualité pour indiquer que l'âge des premières relations sexuelles est plus avancé par rapport à la moyenne ou âge médian des jeunes qui vivent dans le même contexte social. En France, Bajos et collègues (2018) fixent l'entrée dite tardive dans la sexualité à 19 ans. À noter que parmi les migrant.e.s d'Afrique subsaharienne vivant en France qui ont participé à l'enquête quantitative de Marsicano et collègues (2011), les femmes déclarent des rapports sexuels plus tardifs que ceux des hommes (âge médian de 19,1 vs 17,8 ans), situation opposée à celle en Afrique subsaharienne où les femmes ont des rapports sexuels à un plus jeune âge. 


\section{Notes biographiques}

Laura Mellini est sociologue, dr. chargée de recherche et de cours à l'Université de Fribourg (Suisse). Spécialisée sur la santé sexuelle en contexte migratoire, elle fait partie du groupe de recherche GREMISS.Laura.mellini@unifr.ch
Francesca Poglia Mileti est professeure ordinaire à l'Université de Fribourg (Suisse) et spécialiste des migrations et des relations interethniques. Ses travaux récents portent sur la santé sexuelle en contexte migratoire (groupe de recherche GREMISS), la socialisation économique des jeunes, le rapport entre travail et politique.Francesca.poglia@unifr.ch 